\title{
Trading Mechanism Design for Adapting to the Development of Hybrid Electric Energy
}

\author{
Dunnan Liu a , Mingguang Liu ${ }^{b}$, Wenzhuo $\mathrm{Wu}^{\mathrm{c}}$, Ningning Zhaod, Jiarong Hane, \\ Guangyu Qin
}

State Key Laboratory of Alternate Electrical Power System with Renewable Energy Sources(North China Electric Power University), Changping District, Beijing 102206, China

'm18811357955_1@163.com

Keywords: hybrid electric, energy development, trading mechanism

Abstract. Hybrid electric energy (combined cooling heating-power, CCHP) companies has both customer base and power generation resources, therefore, it can be considered as a power user and as a power generation company, which can participate in point-to-point direct purchase of electricity transaction. Based on the characteristics of hybrid electric, this paper designs the commercial operation mode of hybrid electric energy companies, and gives a transaction portfolio for multi-scenario. It helps the companies to adjust their positions according to the power supply and demand in the region, and to participate in the electricity market transactions.

\section{Introduction}

Under the environment of developing low-carbon economy and promoting energy saving and emission reduction in our country, clean energy and distributed energy have become the focus of theoretical research and technological development in the field of energy. Since 2015, China successively promulgated related documents, such as "Opinions on Further Deepening the Reform of Electric Power System [2015] No. 9", the "Measures for the Administration of Combined Heat and Power"," the Guiding Opinions on the Development of "Internet +" Smart Energy Development and Reform [2016] 392 "and so on. These policies encourage the development of distributed power projects and promote the development of hybrid energy with distributed generation. Large-scale application of hybrid energy can become an important force supporting China's energy saving and emission reduction in the future. Also, it can promote electric power reform in our country.

In the market-oriented reform, energy trading shows four characteristics: diversified trading entities, diversified trading products, diversified user selection, and transparency trading information. So design a reasonable and efficient trading mechanism of hybrid energy is the key to optimizing the way of energy development, transmission and utilization, it is also an important means of reflecting the comprehensive value of energy. The trading mechanism for regional CCHP should be able to truly reflect the characteristics of hot and cold electricity trading and the needs of various trading entities. It also should maximize the economic benefits, social benefits and environmental benefits of hybrid energy and improve the profitability of joint ventures, service for hybrid energy's application and development. 


\section{The concept and characteristics of Cooling, Heating, and Power hybrid energy}

\section{Concept}

Combined cooling heating and power (CCHP) system is relative to the traditional centralized power supply system, CCHP is a small-scale energy supply system near the users. It make power generation system build in a decentralized, small-scale layout near the user, so it can effectively reduce both the energy loss during the transmission of electricity, heat, cold and the investment of corresponding transmission system, this system can provide neighboring user with high-quality, high-reliability and clean energy services. This concept is based on energy cascade utilization, and it combines power generation, cooling and heating energy as a system. It can be used as a supplement to the stable supply of power grids and it has the advantages of good reliability and high energy efficiency, small impact on the environment and other characteristics, so it is with good economic and social benefits.

Traditional CCHP mostly depends on single energy sources such as natural gas and coal, which also limits the CCHP's development in geographical, environmental and cost. If we can combine various energy sources with diversified supply modes, and according to the different conditions, design a comprehensive solution, in an effort to make them economically reasonable, it will surely promote the development of CCHP. The appearance of CCHP gives a new idea of building and applying regional cogeneration system. It combines a variety of energy and information flows to form an efficient energy utilization system.

From the point of view of energy structure, hybrid heating and cooling power supply is not based on single energy supply, but instead matches and dispatches multiple energy sources such as natural gas, wind, light, geothermal heat and biomass energy according to different local demand. From the supply point of view, it uses energy station integration technology, not only can realize the collaboration between distributed and centralized supply, it can also choose to connect to the grid or work independently. At the same time, the hybrid heating and cooling power energy network uses intelligent control and cloud computing technology to form an intelligent use of energy, which has supply and demand interaction, orderly configuration, and high energy-saving efficient. Therefore it can maximize energy efficiency.

From the analysis of fuel sources, distributed energy supply systems that use renewable energy such as solar and wind energy as fuel, have the most obviously economic benefits. However, due to the limits of environment, climate, day and night conditions, there are also energy supply unstable defects. Despite the stable and sustainable energy supply of distributed natural gas energy, it subjects to the situation that current domestic gas price is too high, and internet access difficulties, low electricity prices and other factors, so without the government subsidies and support, it will be difficult to operate normally.

In view of this, the establishment of a distributed CCHP system with the distributed natural gas as the core, supplemented by renewable energies such as solar energy, wind energy, biomass energy and geothermal energy, can not only ensure the continuity and stability of energy supply, but also reduce the fuel cost of the system to a certain extent. It is an ideal application of hybrid heat and power energy.

\section{Characteristics}

Distributed CCHP system is a new type of energy system. It combines high-tech and equipment as a whole and realizes cascade utilization of output energy, so it can achieve higher energy efficiency. The development of a distributed CCHP system can play an active role in improving energy efficiency, reducing environmental pollution, developing smart grids, enhancing energy security and optimizing energy structure. It can also provide an effective technological tool for 
dramatically reducing energy consumption in the short term. Under the pressure of energy shortage and energy saving and emission reduction, building a clean, reliable, interactive and efficient distributed hybrid energy system has become an important means to promote the innovation in energy efficient utilization and development of a low-carbon economy.

Natural gas-fueled small or mini-CCHP hybrid system has attracted a great deal of attention in many countries for their diverse forms of energy supply and clean and efficient energy utilization. It has become the focus of the second generation of energy system technologies research. At present, the efficient utilization of clean energy, represented by natural gas's CCHP, is combined with renewable energy, such as ground source heat pump, water storage, ice storage, and thermal storage boilers, to form a CCHP system, and it becomes clean energy industry with rapid development at home and abroad.

The CCHP system includes a variety of micro-power sources, such as distributed photovoltaic power generation, fuel cells, gas internal combustion engines and micro-gas turbines, as well as electric energy storage and heat storage (cold) devices. in which various micro-power sources Power generation characteristics vary, and some micro-power generation characteristics have complementary advantages. Among them, the power generation characteristics of each micro-power source are different, and the power generation characteristics of some micro-power sources have the advantages of complementarity.

It is a comprehensive production, energy system, which is based on the use of energy cascade utilization and scattered around the client. It first uses a primary energy-driven engine power supply, and then recycles waste heat through a variety of waste heat utilization equipment. Ultimately it achieves multi-purpose goals such as higher energy efficiency, lower energy costs, higher energy security and better environmental performance. The energy flow comparison between the distributed CCHP system and the traditional cooling, heating and power supply system is shown in Fig.1

\section{The design for transaction mechanism of combined cooling heating-power system (CCHP)}

\section{Trading mechanism serving for the target of energy saving}

(1)Transaction mode

There are mainly three types of trading methods in the electricity market: bilateral negotiation transaction and centralized matching trading and centralized listing transaction.

1) Bilateral negotiation transaction

The demanding side (buyers) and supplying side (sellers) determine the trading intentions directly (including quantity, price, time of execution and reparation standards for defaulting, etc.) according to the principle of equality and voluntary. Moreover, the contract shall be submitted to the power trading platform within a stipulated time to form a binding transaction contract by checking the quantity and safety of it. 


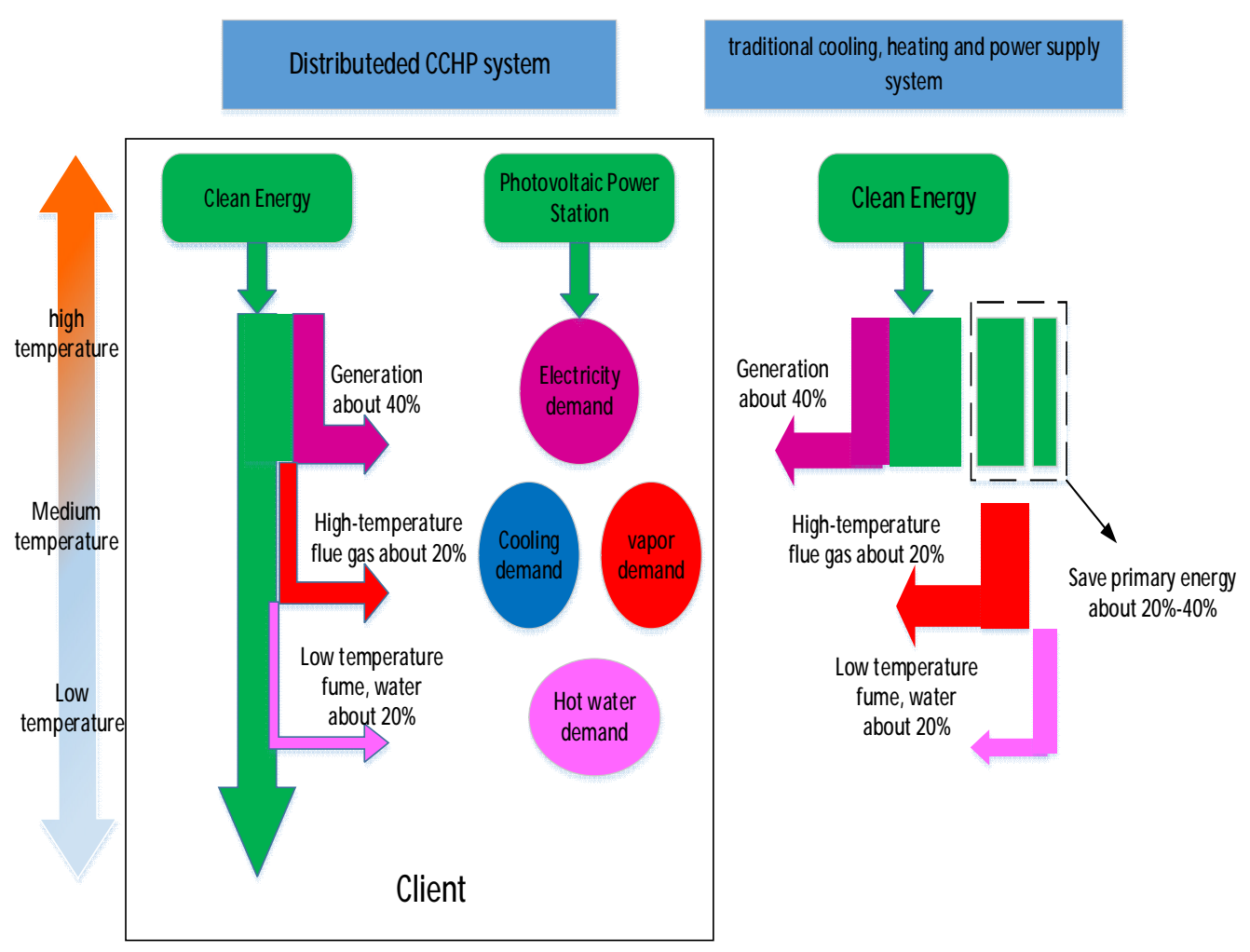

Fig.1. Comparison of different energy supply mode

2) Centralized matching trading

The demanding side (buyers) and supplying side (sellers) declare the transaction demands centrally on the power trading platform, then the platform conducts the transaction matching according to the principle of centralized market clearing forming unconstrained transactions and they will become the restraint ones through the quantity and safety checking by power dispatching center.

Here comes the basic process for a centralized matching transaction.

First, sort the transaction demands of power companies (sellers) and electricity consumers (buyers). Sort the declaring prices of sellers from low to high, in other words, the lower the price is, the more front the corresponding power company and its declaring quantity can be listed; on the contrary, sort the declaring prices of buyers from high to low, that is to say, the higher the price is, the more front the corresponding power company and its declaring quantity can be listed.

Second, match according to the high-low method to achieve market clear. According to the order in the first step, the highest purchasing price is compared with the lowest selling price firstly. If the purchasing price is higher than or equal to the selling price, the transaction is matched, and the transaction price is the mean of the two parties, while the transaction volume is the smaller one declared by the buyer and the seller. And then matching the remaining trading transactions in the same way until all declared purchasing demands (or sales demands) have been traded or the highest purchasing price is below the lowest selling price.

Finally, calculate the clearing capacity and clearing price. The transaction volume is the smaller one declared by the buyers and the sellers. According to the approval pool purchase price of power companies and the bidding price, we can calculate the pair prices of matching transactions in such methods.

The price of biding sellers $=$ approval pool purchase price of power companies + bidding price

The price of biding buyers $=$ bidding price + transmission and distribution price 


\section{3) Centralized listing trading}

The power trading platform promulgates market information according to the determined direct transaction power and transaction price of the cross-provincial and inter-provincial trade, and the sellers (buyers) declare the demanding power quantity through the platform. Then unconstrained transactions can be formed by the platform according to the specified power distribution principle and they will become the restraint one through the quantity and safety checking by power dispatching center.

Here comes the market clearing principles.

First, when the total demanding amount of buyers is less than or equal to the supply, we can satisfy them according to their requirements; otherwise, the electricity shall be distributed in proportion to the demanding quantity declared by the buyers.

Second, when the total supplying amount of sellers is less than or equal to the demand, we can match the transactions according to their declarations; otherwise, the electricity shall be distributed in proportion to the supplying quantity declared by the sellers.

(2) Transaction types classification

Transactions in the power market can be divided into annual transaction, monthly transaction, day-ahead transaction and real-time transaction according to the transaction frequency.

1) Annual transaction

The annual transaction can be conducted in two modes: bilateral negotiation transaction and centralized trading (centralized matching trading and centralized listing trading). The quantity and price of one-handed trading for next year are determined through the transaction.

As a seller, CCHP shall declare the supplying quantity and price for the next year at the end of each year to conduct bilateral or centralized trading with buyers outside its region; and as a buyer, it shall declare the demanding quantity and price for the next year, in order to conduct bilateral or centralized trading with sellers outside its region.

2) Monthly transaction

As the name suggests, monthly transaction is organized once a month. On the basis of long-term transaction contracts divided into twelve pieces, electricity consumers and power generation companies can meet the occasional purchasing (or selling) demands through monthly transactions. Monthly transactions can be organized in two ways: bilateral negotiation and centralized trading (centralized matching trading and centralized listing trading), and out of practical consideration, centralized listing trading can be mainly applied. The monthly bilateral negotiation transactions can be held in the latter several months of this year while the execution time of the centralized trading is the next month.

As a seller, CCHP shall declare the supplying quantity and price for the next month at the end of each month to conduct bilateral or centralized trading with buyers outside its region; and as a buyer, it shall declare the demanding quantity and price for the next month to conduct bilateral or centralized trading with sellers outside its region.

3) Day-ahead transaction

Day-ahead transaction is daily organized. On the basis of monthly transaction contracts divided into thirty pieces, electricity consumers and power generation companies can meet the occasional purchasing (or selling) demands owing to the deviation of load forecasting. Day-ahead transaction can be organized in two ways: bilateral negotiation and centralized listing trading, and its execution time is the next day.

CCHP participates in the day-ahead transaction with day-ahead load forecasting and unit capacity as a reference. As a seller, CCHP shall declare the newly supplying quantity and price for the next 
day before 12:00am each day and complete bilateral or centralized trading with buyers outside its region before 4:00pm; and as a buyer, it shall declare the newly demanding quantity and price for the next day before 10:00am and complete bilateral or centralized trading with sellers outside its region before 4:00pm.

4) Real-time transaction

On the basis of the day-ahead transactions, when there still exists a deviation from the power supply and the electricity demand, real-time transaction in the electricity market will start, namely, searching grid support when demand exceeds supply and seeking delivery allowance when the supply exceeds demand.

(3)Trading mechanism serving for the target of energy saving

On the basis of the existing electricity trading mechanism, the elevated trading mechanism serving for the target of energy saving mainly improves the bidding competitiveness of CCHP by changing the bidding rules and the bidding calculation method and increasing the possibility of transactions of CCHP and clearing prices additionally.

1) Bidding order considering the benefits of emission reduction

When CCHP participates in the electricity market bidding transaction as a power seller, the enterprise shall declare the transaction price and quantity according to its own power generation cost and market supply and demand. The trading center will take the price remaining from the declaring price which cuts off the benefit of emission reduction (The calculation method is as follows: pollutant emission reduction of unit power generation * cost of unit pollutant emissions control) as the bidding price to participate in bidding order. Bidding order considering the benefits of emission reduction effectively raises the clearing prices and improves the possibility of transactions of CCHP.

2) Bidding price considering the energy saving

When the declared prices of sellers which have already considered the benefits of emission reduction of $\mathrm{CCPH}$ system are the same, the order of price and quantity will be formed according to the principle of coal consumption rate each unit priority and CCHP priority. That is, the order forms by the coal consumption rate firstly, and the power generation enterprises with low unit coal consumption rate are ranked in the first table; then each table is sorted by the unit operation mode, and the CCHP is ranked in the first seat. If the declared prices of 2 or more sellers and their environmental benefits, coal consumption rates, and unit operation modes and other parameters are all the same, they will seat on the same chair.

\section{Transaction mechanism of power ancillary service}

(1) The type of ancillary services

The main ancillary service types that the generator set provides to the power grid are: Automatic Generation Control (AGC), Spinning Reserve (Hot standby), Shutdown Spare, Reactive and Voltage Support, Recovery and black-start etc. CCHP is based on natural gas power generation, while gas turbines have the advantages of quick start-stop, fast response and deep peaking. So they are good resources to participate in ancillary services.

(2) Market transaction process

The operation of the market by the trading day, each trading day for a calendar day and it is divided into 48 trading sessions. Each trading session is 30 minutes. While the spot market announces demand, the power trading center publishes the total demand of various auxiliary service markets to market members.

Before 2:00 PM, all generating units with ancillary services license have been declared. Based on the size of the ancillary services market, the power trading center will determine the market 
transaction plan and the market clearing price, which are released before 5:00 pm.

In the real-time scheduling process, if the center deems it necessary, the crew that has obtained the auxiliary service contract may be called to participate in the system adjustment. Within 2 days after the actual operation, the center will liquidate the auxiliary services of the auxiliary service providers and the deviation of the power according to the relevant regulations. It will conduct unified settlement early next month.

(3) Bidding rules

For all kinds of ancillary services, the electricity trading center will sort the quotation of each reporting unit from low to high, and the lowest bidder will get priority. For system safety and reliability considerations, the power trading center may, as appropriate, change the priority of some units. If this happens, the center must give a reasonable analysis and explanation, and keep it for future reference. Co-generation companies can provide their own actual price.

(4)Trading mechanism

Power Trading Center daily organizes FM service, standby operation, alternative standby, cold standby market. Joint cold and hot energy companies can simultaneously declare a number of auxiliary services.

FM service: In any trading session, the difference between the upper limit of adjustment (100\% of rated capacity) declared by the CCHP unit and the base value is the positive chirp capacity of the unit. The difference between the base value and the lower limit of adjustment (0) is the negative FM capacity.

Cold standby: Hot and cold gas turbine can start and stop fast and has low cost, so it is suitable for cold standby. At any trading session, units that have participated in the market bidding but have not been able to access the Internet are used as cold standby for the grid. After receiving the power-on command of the grid dispatch center, the cold-hot gas turbine should be started according to the power-on characteristic curve declared in the data declaration. The unit that is determined to be standby will receive the corresponding standby service fee. Once the unit is called, it will receive extra power generation compensation, which is based on the price of the unit for the corresponding period. For the unit issuing additional reactive power according to the instruction of the dispatch center, it will be compensated accordingly. The compensation price adopts non-linear and incremental pricing method, so it can make up for the increase of costs which is due to the generation of more reactive power.

Voltage reactive support and black start service: CCHP companies provide voltage reactive support and black start services over long-term contracts. According to the operating costs of the FM unit and its contribution to stabilizing the system frequency, the Power Trading Center will pay each FM unit reasonably. When the power system collapses, the CCHP unit is obliged to provide a black start service. Those who make contributions to system recovery will be given a certain reward. Those who cause system accidents or adversely affect system's recovery will be punished. The specific black start protocol and the corresponding incentives and disincentives terms are negotiated by the dispatch center, power grid companies and CCHP companies.

(5) Settlement assessment

In the ancillary services market, the CCHP companies declare the capacity bidding price and electricity bidding price submitted for each service. After the successful bidding, the enterprise will reserve the generating capacity and get the pay of the capacity. In real-time scheduling, if the enterprise is called in real time, then it can get the pay of a certain electricity price. As a result, CCHP companies can get both compensate from the grid companies. 


\section{Other forms of energy trading mechanism}

(1) Thermal trading mechanism

When co-generation of hot and cold mixed energy enterprises cannot meet the demand or when the unit is down or overhauled, they need to purchase thermal power from the thermal power plant or heat from a municipal heating company.

When the direct supply of heat by the municipal heating company, the price of heat implement the government uniform guidance price; when co-generation of hot and cold mixed energy enterprises to energy service providers in the role of thermal power plant to participate in thermal transactions, signed a thermal purchase contract, you can according to supply and demand Situation, heat supply and other indicators, negotiate the price of heat, with the heat of the company's transmission network to complete the heat transfer.

According to the time dimension, thermal trading can be divided into annual (or monthly) trading, trading and real-time trading. The annual (monthly) trading is generally enterprises in the planning overhaul, signed an agreement with the thermal power plant in advance, by their behalf on behalf of heating, heating prices are relatively low; trading is generally based on the next day temperature, economic activity on the heat load Forecast value and supply capacity to make heat trading contracts; real-time trading is a short period of time (such as the next hour) changes in the situation of thermal power supply and demand led to the company and thermal power plant temporary transactions.

(2) Carbon trading mechanism

Co-generation of hot and cold mixed energy enterprises can effectively reduce their carbon emissions, and their carbon savings can be reserved for next year's use as well as participated in carbon trading markets. Relevant trading mechanism implement the relevant provisions of the carbon trading market, the enterprises consider the transaction price of carbon trading market or supply and demand situation, reporting carbon trading volume and price, which declared carbon trading volume cannot exceed the carbon savings enterprises. According to the principle of "matching high and low", match both sides of the carbon deal to reach a deal and obtains carbon trading clearance prices.

\section{Multi-scenario co-generation of hot and cold mixed energy portfolio solutions}

Driven by the most cost-effective target, co-generation of hot and cold mixed energy enterprises will actively adjust the unit operation status, adjust the unit power generation output according to the power demand, actively participate in the electric market transactions, reduce the purchase cost and increase the generation yield. At the same time, the surplus carbon quotas of enterprises are the embodiment of environmental values, and the business benefits can be enhanced through carbon trading. The development of thermal trading depends on the situation of heat supply and demand. During the heating peak or unit troubleshooting period, the enterprises will participate in the heat market transactions. There are many types of services covered in auxiliary services transactions in the electricity market. Enterprises may declare different auxiliary service plans in different periods of time and in different seasons.

At night, when the demand for cooling and heating is low, the gas turbine is in a shutdown state. At this time, the enterprise can be used as a cold standby or a rotating standby. In order to realize Clipping peaks, the enterprise can adjust the running mode of the unit and guiding the user to participate in the demand side response, in peak period of power supply; At any time, both hot and cold motor units can provide black start service, and according to operating conditions, to improve frequency control services for the power grid.

As shown in Fig.2, the multi-scenario hot and cold mixed energy portfolio solution. 


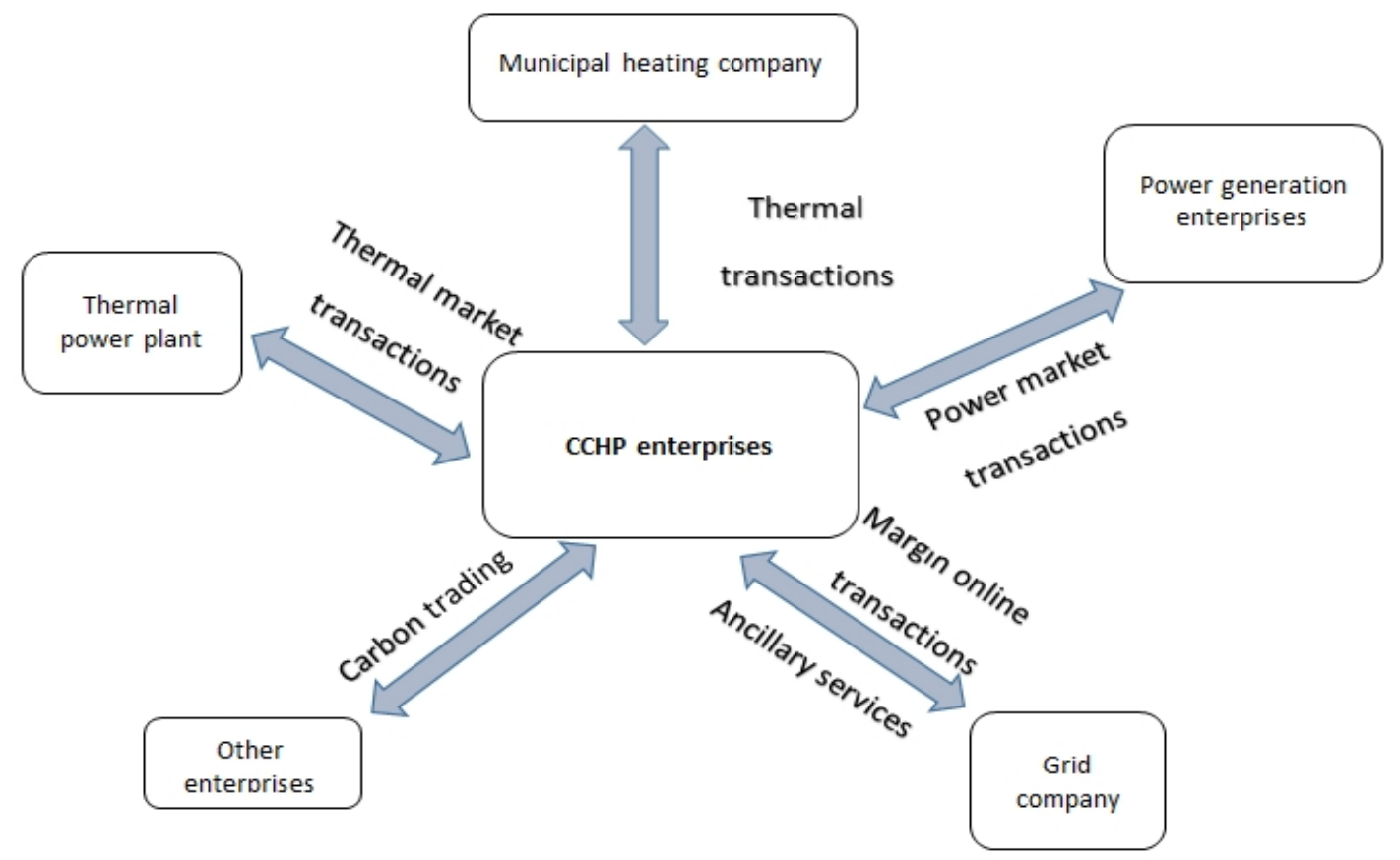

Fig.2. a multi-scenario hot and cold mixed energy portfolio solution.

\section{Conclusions}

Co-generation of hot and cold mixed energy can effectively improve the energy utilization efficiency, ease the impact of distributed generation on the large power grid and promote the development of clean energy such as distributed photovoltaic. For the power industry, co-generation enterprises, users and the community as a whole, can bring huge economic benefits, has good potential for development. In this paper, we design the transaction mechanism of such energy system based on its own characteristics, including the power trading mechanism that serve the goal of energy saving and emission reduction, power ancillary services transaction mechanism and other energy's trading mechanisms. And put forward a multi-scenario hot and cold mixed energy portfolio solution, thereby enhancing the enthusiasm of enterprises to invest. In this way, such resources in China's energy-saving emission reduction and low-carbon economy in the process of development and optimization play an catalytic role.

\section{References}

[1] Duan Shaohui, Wang Wei, Liu Zhongsheng, et al.Optimization scheduling of microgrid system with cogeneration for photovoltaic power generation [J]. Journal of Power Systems and Automation, 2013,8 (4): 150-155

[2] ZHANG Tao, ZHU Tong, GAO Nai-ping, et al.Study on optimal design and multi-index comprehensive evaluation of distributed thermal power system [J].

[3] Wang Cheng, Xu Yancai, Wei Qinglai, et al.Analysis of business model and operation strategy of intelligent community [J] .Power System Protection and Control, 2015,3 (6): 147-154 